\title{
Short-range correlation effects on the plasmons in cylindrical tubules
}

\author{
B. Tanatar \\ Department of Physics, Bilkent University, Bilkent, 06533 Ankara, Turkey
}

(Received 21 August 1996)

\begin{abstract}
We study the collective excitation of single and coaxial cylindrical tubules in the very low density regime. Exchange-correlation effects neglected in the random-phase approximation are shown to be important for plasmon modes. We also calculate the spectral weight function $\operatorname{Im}[1 / \varepsilon(q, \omega)]$ to investigate the collective modes as measured in electron energy-loss spectroscopy experiments. [S0163-1829(97)06804-5]
\end{abstract}

The recent developments in the synthesis techniques have led to the fabrication of hollow tubules of graphitic materials. ${ }^{1}$ A graphene tubule is essentially a graphitic sheet rolled up in the cylindrical form. Charge carriers may be introduced onto the tubules in a controlled manner through intercalation $^{2}$ as in the case of graphite intercalated compounds. ${ }^{3}$ The typical radius of such cylindrical tubules is several nanometers, which results in a one-dimensional character of the motion of these carriers. Experimentally it has been found that the synthesized tubules can be open ended. ${ }^{4}$ Metallic or semiconducting behavior of single tubules is predicted. ${ }^{5}$ From a theoretical point of view carbon-based microstructures offer opportunities to study the effects of dimensionality on the collective excitations. ${ }^{6}$ Moreover, the carbon nanotubules have caused considerable excitement and activity because of possible device applications. ${ }^{7}$

The charge carriers on a tubule may be modeled by a quasi-one-dimensional (Q1D) electron-gas as a first step towards the full understanding of complicated excitation spectra of graphitic materials. The elementary excitations in cylindrical tubules were studied by Lin and Shung ${ }^{8}$ using the random-phase approximation (RPA). Longe and Bose ${ }^{9}$ have presented semiclassical calculations for metallic graphene tubules. A more systematic study of the excitation spectrum of multishell fullerenes and coaxial nanotubules appeared recently. ${ }^{6}$ A microscopic approach utilizing the symmetry properties is also reported for carbon fibers. ${ }^{10}$ Band-structure effects have recently been considered. ${ }^{11}$ The plasma modes in coaxial carbon tubules have been observed experimentally by means of electron energy-loss spectroscopy. ${ }^{12}$

Our aim in this paper is to examine the collective excitations of very low density electrons on single and coaxial tubules. We include the short-range correlation effects through the local-field corrections. A similar approach was taken by Miesenböck and Tosi ${ }^{13}$ in their study of layered metal intercalated graphite. The previous works on the collective excitations of graphene tubules have used the RPA, which takes into account dynamic screening in the electrongas but does not include the corrections due to exchangecorrelation (xc) effects. The local-field theory ${ }^{14}$ of Singwi et al. (STLS) uses the static pair-distribution function to approximate the short-range electronic correlations. It has been noted that corrections to the RPA become more important in one- and two-dimensional systems than that in the bulk. Systems with finite number of particles also show strong dependence on the many-body effects. ${ }^{15}$ It has been found, for instance, that the xc effect on the plasmon dispersion decreases with increasing cluster size.

A free-electron gas model for cylindrical tubules was developed by several researchers. ${ }^{8,16,17}$ The one-dimensional (1D) nature of the system has similarities to other quasi-onedimensional electron-gas models commonly used to describe the semiconducting quantum wires. ${ }^{18} \mathrm{~A}$ notable feature of the hollow tubule model is that the angular momentum quantum number $L$ completely decouples the intrasubband ( $L=0)$ and intersubband $(L \neq 0)$ excitations from each other.

The collective excitations of a single tubule are determined from the zeros of the dielectric function $\varepsilon(q, \omega ; L)=1-V(q ; L)[1-G(q ; L)] \chi(q, \omega ; L)$, where the Coulomb interaction between electrons on a tubule is ${ }^{19}$ $V(q ; L)=\left(4 \pi e^{2} / \epsilon_{0}\right) I_{L}(q R) K_{L}(q R)$, in which $I_{L}(x)$ and $K_{L}(x)$ are the modified Bessel functions of the first and second kind, respectively. The local-field factor $G(q ; L)$ modifies the bare Coulomb interaction by describing the xc effects neglected in the RPA. The density response function $\chi(q, \omega ; L)$ appropriate for electrons on a tubule is derived by several authors. ${ }^{8,16,17}$ It has contributions from occupied subbands $(l=0, \pm 1, \pm 2, \ldots)$, thus giving rise to a rich excitation spectrum for the collective modes. In the following we shall concentrate on the most energetic plasmon mode, since the oscillator strength is dominated by this mode. ${ }^{8}$ The localfield factor $G(q ; L)$ is calculated using the $1 \mathrm{D}$ version of the STLS scheme ${ }^{14}$ self-consistently with the static structure factor $S(q)$ as obtained from the frequency integral of the fluctuation-disspation theorem.

We first discuss the results of our calculations for a single tubule. For illustration purposes we take the radius of the tubule $R=11 \AA$, the effective mass of the electrons $m^{*}=m_{e} / 4$, and the background dielectric constant $\epsilon_{0}=2.4$, so that a graphene tubule is closely described. We assume that the electrons are introduced through intercalation. The linear electron density is chosen to be $n_{1 \mathrm{D}} a_{B}^{*}=1 /\left(2 r_{s}\right)$ $=0.5$, where $a_{B}^{*}$ is the effective Bohr radius and $r_{s}$ is the dimensionless electron-gas coupling parameter. This corresponds to a sheet density of $n_{2 \mathrm{D}}=n_{1 \mathrm{D}} /(2 \pi R) \approx$ $1.45 \times 10^{13} \mathrm{~cm}^{-2}$, low enough to make the short-range correlation effects appreciable. For the above parameters we find the Fermi energy to be $E_{F}=0.14 \mathrm{eV}$, so that only the lowest three subbands are occupied, viz., $l=0, \pm 1$. 

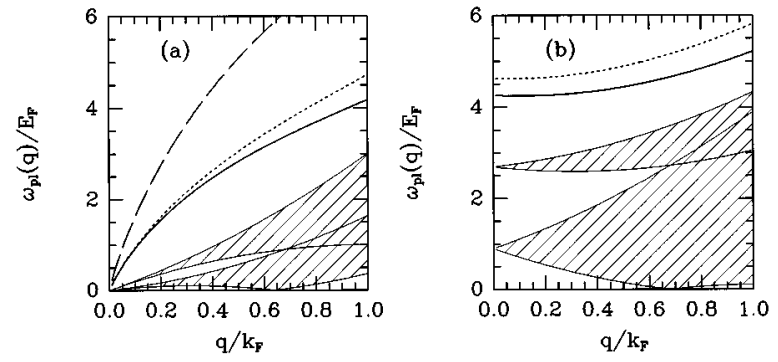

FIG. 1. (a) The intrasubband $(L=0)$ and (b) the intersubband $(L=1)$ plasmon dispersions for a single tubule. Solid and dotted lines are with and without local-field factors, respectively. The dashed line represents the long-wavelength approximation.

We show the dispersion of $L=0$ intrasubband plasmons for a single graphene tubule in Fig. 1(a). The solid and dotted lines correspond to $\omega_{\mathrm{pl}}(q)$ with and without (RPA) the shortrange correlations, respectively. It is observed that the localfield effects on $\omega_{\mathrm{pl}}(q)$ become noticeable for $q \geqslant 0.3 k_{F}$. We also show (dashed line) the approximate plasmon dispersion obtained by long-wavelength expansion. The intersubband $(L=1)$ plasmon dispersions are displayed for the same system in Fig. 1(b). The short-range correlation effects move the dispersion curve down roughly by a constant amount for the range of wave vectors of interest. The electron-energy-loss spectroscopy (EELS) experiments ${ }^{20}$ on multilayer carbon tubules reveal information on the collective excitations. We calculate the quantity $\operatorname{Im}[-1 / \varepsilon(q, \omega ; L)]$ measured in EELS experiments. Figures 2(a) and 2(b) show the spectra at $q=0.5 k_{F}$ for $L=0$ and $L=1$ plasmons, respectively. The solid and dotted lines are obtained with and without the local-field corrections. To identify the collective modes we have introduced a small broadening parameter $\Gamma=0.01 E_{F}$ along the lines discussed by Lin et al. ${ }^{11}$ In both cases the plasmon peak is clearly identified. The short-range correlation effects described by the local-field corrections move the peak position towards the low-energy side. The spectral weight due to the particle-hole continuum is typically small compared to that of plasmons.

We now consider two coaxial tubules with radii $R_{1}$ and $R_{2}\left(R_{2}>R_{1}\right)$. Assuming that intertubule hopping or tunneling is negligible, the Coulomb interaction between two electrons is given by $V_{i j}(q ; L)=\left(4 \pi e^{2} / \epsilon_{0}\right) I_{L}\left(q R_{<}\right) K_{L}\left(q R_{>}\right)$, where $R_{<}\left(R_{>}\right)$denotes the smaller (larger) of $R_{1}$ and $R_{2}$. Here $i$ and $j(i, j=1,2)$ label the tubules. The coupled plas-
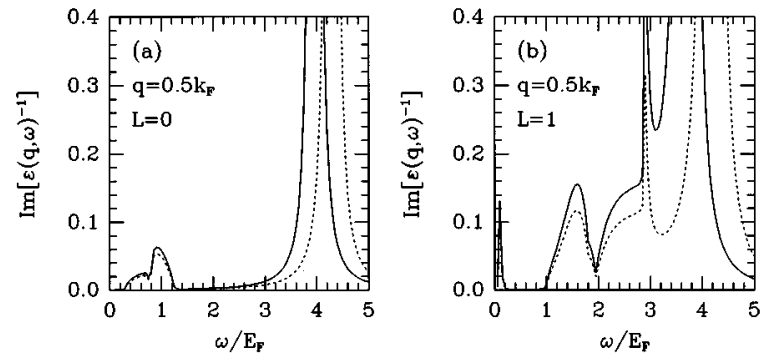

FIG. 2. (a) The intrasubband and (b) intersubband spectral weight function $\operatorname{Im}[1 / \varepsilon(q, \omega)]$ for a single tubule at $q=0.5 k_{F}$. The solid and dotted lines are with and without local-field corrections, respectively.
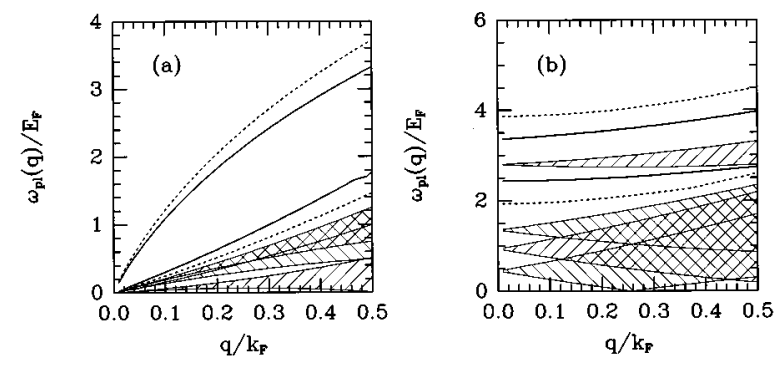

FIG. 3. (a) The intrasubband ( $L=0$ ) and (b) the intersubband ( $L=1$ ) plasmon dispersions for two coaxial tubules. Solid and dotted lines are with and without local-field factors, respectively.

mon modes in coaxial tubules is obtained from the solution of $\operatorname{det}|\varepsilon(q, \omega ; L)|=0$, where the elements of the dielectric matrix are given by $\varepsilon_{i j}(q, \omega ; L)=\delta_{i j}$ $-V_{i j}(q ; L)\left[1-G_{i j}(q ; L)\right] \chi_{j}(q, \omega ; L)$. In the above expression, we may use the generalization ${ }^{21}$ of the STLS method to a two-component system to include intratubule and intertubule local-field corrections $G_{i j}(q ; L)$. Setting $G_{i j}=0$ one recovers the RPA expression for $\varepsilon_{i j}(q, \omega ; L) \cdot \chi_{i}(q, \omega ; L)$ is the response function of the $i$ th tubule. The fully self-consistent determination of intratubule and intertubule local-field corrections is computationally more demanding. We further employ the Hubbard approximation (HA) to the local-field corrections that use the Hartree-Fock static structure factor as input. Approximately, one obtains

$$
G_{i j}^{\mathrm{HA}}(q ; L)=\frac{1}{2} \frac{\left.V_{i i}\left(\sqrt{q^{2}+k_{F}^{2}} ;\right)\right)}{V_{i i}(q ; L)} \delta_{i j} .
$$

The Hubbard approximation takes exchange into account but neglects Coulomb correlations in calculating the local-field correction between the particles within the same tubule. Since the intertubule interaction is typically ${ }^{8}$ smaller than the intratubule interaction at low $q$, RPA could be used in the former case. In the following calculations we take $R_{1}=11$ $\AA$ and $R_{2}=14.4 \AA$, and assume that both tubules are at the same linear electron density $n_{1 \mathrm{D}}$. This implies that the areal density of electrons on each tubule will be different. In the examples below we take $r_{s}^{(1)}=1$ and $r_{s}^{(2)}=0.61$. We assume that the charge carriers may be introduced onto the tubules by means of intercalation at the desired densities.

The coupled intrasubband plasmon modes $(L=0)$ in a two-coaxial tubule system are shown in Fig. 3(a). As before, we only exhibit the two most energetic plasmon branches since the other modes have much weaker oscillator strength. The solid and dotted lines indicate $\omega_{\mathrm{pl}}(q)$ with and without the local-field factors, respectively. In the case of RPA $\left(G_{i j}=0\right)$ there exist two plasmon modes corresponding to the in and out-of phase oscillations of the charges. The effect of short-range correlations on these modes is such that the in-phase mode is softened [upper curves in Fig. 3(a)] whereas the out-of-phase mode is hardened [lower curves in Fig. 3(a)]. It is also noted that the out-of phase mode is truly acoustic in character, and is affected by the local-field effects more than the in-phase mode. Figure 3(b) displays the inter- 
subband plasmon modes $(L=1)$. We observe that the optical plasmon modes are affected by the short-range correlations for densities of $r_{s} \sim 1$.

In summary, we have considered the collective excitations in single and coaxial graphene tubules. The charge carriers are assumed to be introduced by intercalation. The lowdensity electron-gas formed on the surface of tubules is studied beyond the usually employed RPA. The local-field effects describing correlations beyond the simple RPA seem to be very important for low densities. It is found that the shortrange correlation effects modify both the intrasubband and intersubband plasmon dispersions significantly. More realistic calculations, such as the time-dependent local-density approximation approach, ${ }^{15}$ incorporating the xc effects in the single-particle spectra would be needed to determine critically the importance of many-body effects. Increased advances in semiconductor technology also make possible the fabrication of quantum-well wires in a tubulelike structure. In particular, quantum wires of GaAs surrounded by $\mathrm{Ga}_{x} \mathrm{Al}_{1-x} \mathrm{As}$ with very small dimensions have been achieved. ${ }^{22}$ Our calculations may be applied to the analysis of such systems, as well as the recently discussed drag effect in coaxial cylindrical quantum wells. ${ }^{23}$

This work is partially supported by the Scientific and Technical Research Council of Turkey (TUBITAK) under Grant No. TBAG-AY/77. We thank Professor S. Çıracı for useful discussions.
${ }^{1}$ S. Iijima, Nature 354, 56 (1991); T. W. Ebbesen and P. M. Ajayan, ibid. 358, 220 (1992).

${ }^{2}$ O. Zou et al., Science 263, 1744 (1994).

${ }^{3}$ M. S. Dresselhaus, Intercalation in Layered Materials (Plenum, New York, 1987).

${ }^{4}$ S. Iijima, P. M. Ajayan, and T. Ichihashi, Phys. Rev. Lett. 69, 3100 (1992).

${ }^{5}$ J. W. Mintmire, B. I. Dunlap, and C. T. White, Phys. Rev. Lett. 68, 631 (1992); N. Hamada, S. I. Sawada, and A. Oshiyama, ibid. 68, 1579 (1992); R. Saito et al., Appl. Phys. Lett. 60, 2204 (1992); H. Ajiki and T. Ando, J. Phys. Soc. Jpn. 62, 1255 (1993).

${ }^{6}$ C. Yannouleas, E. N. Bogachek, and U. Landman, Phys. Rev. B 53, 10225 (1996).

${ }^{7}$ For a popular account see M. Dresselhaus, Physics World 9, 18 (1996).

${ }^{8}$ M. F. Lin and K. W.-K. Shung, Phys. Rev. B 47, 6617 (1993).

${ }^{9}$ P. Longe and S. M. Bose, Phys. Rev. B 48, 18239 (1993).

${ }^{10}$ M. S. Dresselhaus, G. Dresselhaus, and R. Saito, Phys. Rev. B 45, 6234 (1992).

${ }^{11}$ M. F. Lin et al., Phys. Rev. B 53, 15493 (1996).
${ }^{12}$ L. A. Bursill et al., Phys. Rev. B 49, 2882 (1994); P. M. Ajayan, S. Iijima, and T. Ichihashi, ibid. 47, 6859 (1993); R. Kuzuo, M. Terauchi, and M. Tanaka, Jpn. J. Appl. Phys. 31, L1484 (1992).

${ }^{13}$ H. M. Miesenböck and M. P. Tosi, Z. Phys. B 78, 255 (1990).

${ }^{14}$ K. S. Singwi et al., Phys. Rev. 179, 589 (1968).

${ }^{15}$ C. Yannouleas, E. Vigezzi, and R. A. Broglia, Phys. Rev. B 47, 9849 (1993); C. Yannouleas et al., Phys. Rev. Lett. 63, 255 (1989); C. Yannouleas, F. Catara, and N. Van Giai, ibid. 50, 7977 (1994); 51, 4569 (1995).

${ }^{16}$ H. Chen, Y. Zhu, and S. Zhou, Phys. Rev. B 36, 8189 (1987); Y. Zhu, F. Huang, X. Xiong, and S. Zhou, ibid. 37, 8992 (1988).

${ }^{17}$ O. Sato et al., Phys. Rev. B 48, 1947 (1993).

${ }^{18}$ Q. P. Li and S. Das Sarma, Phys. Rev. B 43, 11768 (1991); A. Gold and A. Ghazali, ibid. 41, 7626 (1990).

${ }^{19}$ J. D. Jackson, Classical Electrodynamics, 2nd ed. (Wiley, New York, 1975).

${ }^{20}$ P. M. Ajayan, S. Iijima, and T. Ichihashi, Phys. Rev. B 47, 6859 (1993); L. A. Bursill et al., ibid. 49, 2882 (1994).

${ }^{21}$ A. Sjölander and J. Stott, Phys. Rev. B 5, 2109 (1972).

${ }^{22}$ P. M. Petroff et al., Appl. Phys. Lett. 41, 635 (1982).

${ }^{23}$ G. Qin, J. Phys. Condens. Matter 7, 9785 (1995). 